B-10-2020

\title{
AJUSTE DEL CÁLCULO DE LAS DOTACIONES DE LOS HIDRANTES A LA GEOMETRÍA DE LAS PARCELAS
}

Goicoechea Iriarte, F.(1) (P), Ederra Gil, I.(2), Puig Arrastia, J., (3), Campo-Bescós, M.A. (4), Pérez Garciandia, D. (5) Javiera Cirauqui Lacarra (6)

\footnotetext{
1.Estudiante, Universidad Pública de Navarra, Pamplona, fermingoico.i@gmail.com

2.Directora de explotación de la Ampliación de la $1^{\text {a }}$ Fase de la zona regable del Canal de Navarra, INTIA, Villava, Navarra. iederra@intiasa.es

3.Subdirector de INTIA, Villava, Navarra. jpuig@intiasa.es

Profesor, Universidad Pública de Navarra, Departamento Ingeniería, Pamplona, miguel.campo@unavarra.es

5.Técnico de explotación de la Ampliación de la $1^{\text {a }}$ Fase de la zona regable del Canal de Navarra, INTIA, Villava, Navarra. dperez@intiasa.es

5.Operadora de explotación de la Ampliación de la $1^{\text {a }}$ Fase de la zona regable del Canal de Navarra, INTIA, Villava, Navarra. jcirauqui@intiasa.es
}

\section{Resumen}

Este estudio se desarrolla en el sector de riego XXIV de la Ampliación de la $1^{a}$ Fase del Canal de Navarra (España). Dicha ampliación forma parte del sistema Itoiz - Canal de Navarra, que recorre la comunidad foral abasteciendo de agua de boca y de riego a una multitud de localidades. Una característica que define a dicho sector es la complicada geometría de sus unidades de riego. El proceso de construcción de esta ampliación sigue el siguiente orden, en primer lugar, el dimensionamiento de los hidrantes que suministrarán caudal a las parcelas y posteriormente la instalación de dichas parcelas. En el sector XXIV, una vez ejecutadas las instalaciones de parcela se ha comprobado que la demanda de caudal de ciertas unidades de riego (UURR) es superior a las dotaciones asignadas previamente en el diseño de la red general. Esto provoca un descenso en la uniformidad y eficiencia del riego en la parcela. En el presente trabajo se plantea relacionar la demanda de caudal de las UURR con su geometría, de forma que la dotación del hidrante se ajuste a la demanda real. La geometría de la unidad de riego se relaciona a su vez con la proporción de aspersores sectoriales, de mayor pluviometría que los circulares, instalados en el contorno de la unidad de riego. Para ello, se calcula un coeficiente de mayoración por efecto borde para una muestra representativa de diseños de instalaciones de riego por aspersión, y se vincula con un parámetro de geometría obtenido para cada una de estas UURR. De esta forma, se obtiene una fórmula aplicable a cualquier UR cuya dotación se desee conocer. 


\section{Introducción y objetivos del trabajo}

Este trabajo se desarrolla en la Comunidad Foral de Navarra (España), donde se sitúa el embalse de Itoiz, construido para abastecer de agua el Canal de Navarra. El Canal de Navarra es un proyecto cuya financiación, construcción y explotación es compartida entre el Gobierno de España y el Gobierno de Navarra con el objetivo, entre otros, de realizar una transformación en regadío de 53.125 hectáreas. Para ello crearon, en enero del año 2000 la sociedad estatal "Canal de Navarra S.A." con una participación del 60\% Estado y 40\% Gobierno de Navarra (CANASA, 2000).

En la actualidad se han puesto en riego, a través de concesiones (contratos entre la empresa pública INTIA como concedente y las empresas privadas AGUACANAL y AGUAS DE NAVARRA como concesionarios), en una primera fase, 22.404 hectáreas y 6.361 hectáreas más en su ampliación, repartidas en 25 sectores de riego (Sectores I, II.1, II.2, III, IV.1, IV.2, IV.3, IV.4, IV.5, V; VI, VII, VIII, IX; X, XXIIArga 1A, XXII-Arga 1B, XXII-Arga 2, XXII-Arga 3, XXII-Arga 5, XXII-Arga 6, XXIII, XXIV; XXV y XXVI-Ega 1)

Dentro de la zona regable del Canal de Navarra, el estudio se realiza en la Ampliación de la $1^{\text {a }}$ Fase del Canal de Navarra, concretamente en el sector XXIV. Una característica peculiar del sector XXIV con respecto al resto de los sectores de riego es la geometría irregular de sus $U_{U R R^{1}}$. Se trata de una zona con una topografía complicada y con muchas zonas de alto valor ambiental, por lo que, en la fase de concentración parcelaria no fue posible que las UURR tuviesen las habituales formas cuadradas o rectangulares.

Como ya se ha comentado, la Ampliación de la $1^{\text {a }}$ Fase del Canal de navarra se ha desarrollado a través de un sistema concesional. En esta zona regable, la sociedad concesionaria es Aguas de Navarra S.L., participada por OHL (65\%) y Aguas de Barcelona (35\%). Esta empresa financia y construye las infraestructuras de Interés General, y las explota durante 30 años. Las infraestructuras de Interés General son aquellas necesarias para transportar y distribuir el agua desde el Canal hasta las UURR de cada regante, incluyendo los hidrantes, a los que se les asigna una dotación estimada según la superficie de la UR.

La empresa concesionaria explota y mantiene dichas infraestructuras a lo largo de 30 años, tiempo durante el cual, el Gobierno de Navarra a través del Instituto Navarro de Tecnologías e Infraestructuras Agroalimentarias S.A (INTIA) como empresa concedente, abona una retribución mensual por el coste de construcción de dichas infraestructuras, que se une a la retribución abonada por los regantes en base al canon de explotación.

Con el fin de asegurarse de que la empresa concesionaria realiza su trabajo correctamente y otorga un servicio óptimo a los regantes, el Gobierno de Navarra aplica una serie de deducciones condicionadas al adecuado funcionamiento de las infraestructuras, basadas en unos indicadores descritos en el Pliego de Preinscripciones Técnicas para la Explotación (PPTE) del contrato.

Una vez han sido dimensionadas y construidas las redes de interés general por la empresa concesionaria, los propietarios de las UURR contratan a su elección a un instalador particular que diseña y construye su instalación de riego en parcela. Estos instaladores deberán atenerse al caudal de consigna que ha sido asignado

\footnotetext{
${ }^{1}$ Unidad de riego: se define como una superficie de un valor igual o superior a 5 hectáreas que permite un diseño racional de las instalaciones de riego en parcela (Ley Foral 1/2002, de 7 de marzo de Infraestructuras Agrícolas)
} 
como dotación del hidrante de su UR. En el diseño de las instalaciones de riego por aspersión con un marco de riego $15 \times 18 \mathrm{~m}$ (15 metros entre líneas y 18 metros entre los aspersores de la línea) habitual en estos lares, se utilizan dos tipos de aspersores: circulares y sectoriales. Los circulares con boquillas de 4,4 y $2,4 \mathrm{~mm}$ emiten un caudal de $1.800 \mathrm{l} / \mathrm{h}$ a una presión en boquilla de $35 \mathrm{mca}$, lo que supone una pluviometría de $6,67 \mathrm{l} /\left(\mathrm{m}^{2} \times \mathrm{h}\right)$. Los aspersores sectoriales se instalan en los bordes de la UR y aplican una pluviometría de $8,15 \mathrm{l} /\left(\mathrm{m}^{2} \times \mathrm{h}\right)$, ya que se estima que disponen de una boquilla de $4 \mathrm{~mm}$ y de un caudal de $1.100 \mathrm{l} / \mathrm{h}$ a una presión en boquilla de $35 \mathrm{mca}$. Se debe tener en cuenta que la superficie regada por cada uno de estos aspersores sectoriales es la mitad que en los circulares.

Siguiendo este proceso y al igual que en el resto de los sectores de riego, en el sector XXIV el concesionario, con la supervisión de INTIA, ha dimensionado y construido las redes de interés general calculando una dotación para los hidrantes de este sector en función de la superficie de las UURR.

El problema ha surgido cuando una vez construidas las infraestructuras de interés general y las instalaciones de riego en parcela, se ha constatado que el caudal demandado es superior al que son capaces de suministrar los hidrantes.

Como ya se ha comentado, el sector XXIV es peculiar en cuanto a la geometría irregular de sus UURR, y este trabajo trata de vincular esta peculiaridad con los mayores requerimientos para regar eficientemente estas UURR con morfologías que se salen de lo habitual.

Por tanto, el objetivo final de este estudio es:

Establecer una fórmula de cálculo de las dotaciones de los hidrantes que considere la geometría de las unidades de riego además de su superficie.

\section{Descripción}

En primer lugar, se detallan las características de la zona de estudio, para pasar posteriormente a desarrollar la metodología y los materiales utilizados.

\subsection{Zona de estudio}

El análisis realizado en este trabajo se centra en el sector XXIV (Figura 1), que transforma de secano a regadío una superficie de 1.713 hectáreas y un total de 283 hidrantes que suministran agua a UURR, en algunos casos con inusuales y complicadas geometrías, de los municipios de Larraga y Mendigorría. Hoy, tras dos años en explotación, se encuentran en servicio 199 hidrantes (el 68\% del sector).

Además, se analizarán UURR del sector XXII-Arga 5 (Figura 2) para asegurar que la fórmula final se adapta a todo tipo de UURR. Este sector pertenece, al igual que el sector XXIV, a la zona regable de la Ampliación de la $1^{\text {a }}$ Fase del Canal de Navarra. La forma de sus UURR corresponde a la habitual tras los procesos de concentración parcelaria obligatorios en estas actuaciones, con una geometría altamente regular y los caudales que requieren no se desvían de los suministrados por los hidrantes. El sector XXII-Arga 5, de 693 hectáreas y 110 hidrantes, es el resultado de la modernización del regadío tradicional del municipio navarro de Funes. 


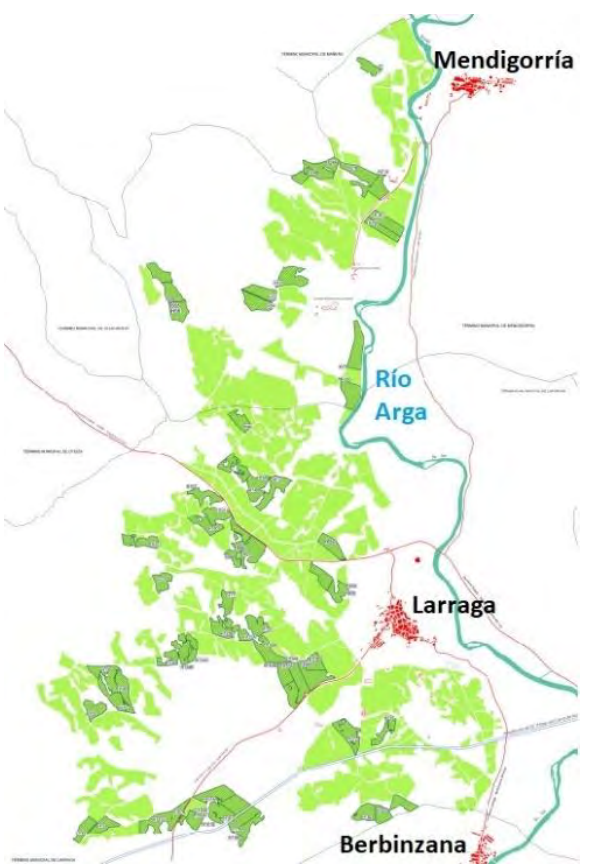

Figura 1: Sector XXIV de la Ampliación de la $1^{\text {a }}$ Fase del Canal de Navarra. En un tono más oscuro, las Unidades de Riego estudiadas en el proyecto

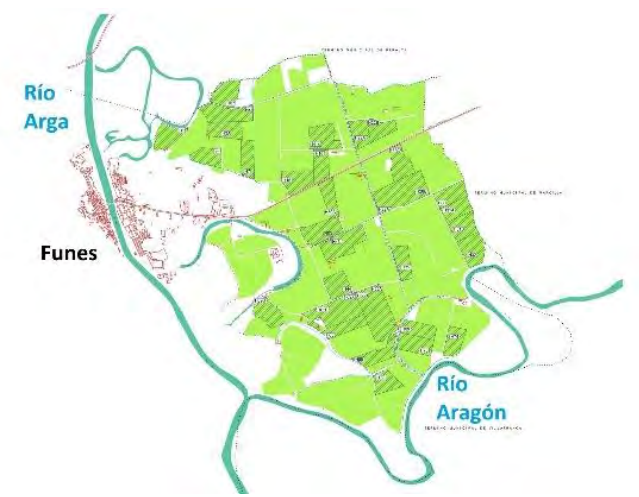

Figura 2: Sector XXII-Arga 5 de la Ampliación de la $1^{\text {a }}$ Fase del Canal de Navarra. En un tono más oscuro, las Unidades de Riego estudiadas en el proyecto

\subsection{Nuevos coeficientes de efecto borde}

En el cálculo de las dotaciones de los hidrantes de los sectores construidos de la Ampliación de la $1^{\text {a }}$ Fase del Canal de Navarra, INTIA utiliza una fórmula empírica (1) que ha desarrollado en base a 35 años de experiencia y a alrededor de 70.000 hectáreas transformadas o modernizadas en Navarra a sus espaldas:

$$
Q_{i}(1 / s)=\left(S_{i} / 7\right) \times(6,67 / 3.600) \times 1,075
$$

\section{Donde:}

- $S_{\mathrm{i}}$ : Superficie de cada Unidad de Riego (UR) en $\mathrm{m}^{2}$.

- 7: $\mathrm{N} .{ }^{\circ}$ de sectores en que de forma general se dividen las unidades de riego. EI motivo por el que se dividen en 7 sectores es poder regar la UR evitando las horas del día con más viento, además de que haya un margen de tiempo por 
posibles problemas y averías en las condiciones de máxima necesidad del cultivo. En las condiciones de mayores necesidades de riego (con maíz en el mes de julio) son necesarios $54,78 \mathrm{l} / \mathrm{m}^{2}$ semanales. Con una pluviometría de 6,67 $\mathrm{l} /\left(\mathrm{m}^{2} \mathrm{xhora}\right)$, son necesarias $8,22 \mathrm{~h}$ de riego por semana en cada sector.

- 6,67: Pluviometría, en $1 /\left(\mathrm{m}^{2} \times\right.$ hora $)$ de los aspersores circulares. "Ya explicado en la introducción"

- 1,075: Coeficiente de mayoración de la pluviometría por el efecto borde, estimando que el porcentaje de aspersores sectoriales, con una pluviometría de $8,15 \mathrm{l} /\left(\mathrm{m}^{2} \times\right.$ hora $)$, que se instala en una UR se encuentra entre el 30 y el $35 \%$, adimensional.

Como se puede observar, esta ecuación contempla únicamente una variable, que es la superficie de la UR a la que se va a suministrar el caudal. Con el fin de que el cálculo de la dotación del hidrante se ajuste de forma más precisa a los requerimientos, se analiza el coeficiente de mayoración por efecto borde utilizado. Este coeficiente se basa en que todos los sectores de una UR regular van a tener el mismo porcentaje de aspersores sectoriales respecto al total. Esto no es así, fundamentalmente en UURR irregulares.

Por este motivo se analiza, en primer lugar, el número de aspersores circulares y sectoriales y, en segundo lugar, la pluviometría de los aspersores sectoriales de 78 UURR diseñadas por 4 profesionales diferentes. Se calcula un nuevo coeficiente de mayoración de efecto borde para cada una de ellas. De estas 78 UURR analizadas, 47 pertenecen al sector XXIV, que se han visto afectadas por el problema ya mencionado de desajuste de caudales, y 31 al sector XXII-Arga 5, que se utiliza como control, ya que en su puesta en marcha no surgió ningún problema.

Para calcular los nuevos coeficientes de mayoración por efecto borde (EfB) de las UURR analizadas se utiliza la siguiente fórmula (2).

$$
E f B=\frac{\left(\left(\frac{6,67}{3.600}\right) \times(1-\% \text { sect })\right)+\left(\left(\frac{9,78}{3.600}\right) \times(\% \text { sect })\right)}{(6,67 / 3.600)}
$$

Donde:

- EfBI: Coeficiente de mayoración por efecto borde, adimensional.

- 6,67: Pluviometría de los aspersores circulares en $\mathrm{I} /\left(\mathrm{m}^{2} \times\right.$ hora $)$

- 9,78: Nueva pluviometría para los aspersores sectoriales en $1 /\left(m^{2} \times h o r a\right)$. Se ha comprobado que la práctica de los instaladores locales es aumentar la pluviometría del contorno de la UR en un $20 \%$, con objeto de compensar las pérdidas de agua por efecto del viento.

- \% sect: Porcentaje de aspersores sectoriales en cada una de las 78 UR analizadas

\subsection{Análisis de la geometría}

Una vez se han calculado los coeficientes de efecto borde, se pasa a analizar la geometría de estas 78 UURR. El fin de este apartado es buscar la 
manera de parametrizar la forma de las UURR. Se trata de calcular un índice para medir la geometría, que pueda ser obtenido antes de conocer la instalación interior de la UR, y que represente la proporción de aspersores sectoriales sobre circulares con la que se instalará la UR en el futuro. En consecuencia, se opta por el siguiente índice geométrico que relaciona el perímetro con el área de la UR (3).

$$
I G=(P \times 100) / S
$$

Donde:

- IG: Índice Geométrico utilizado para parametrizar la forma de las UR, adimensional.

- P: Perímetro de la UR (en m). Cuando una UR tenga en su interior una o más islas de secano (con por ejemplo valores naturales de obligada conservación), sus perímetros se deben sumar al de la UR, ya que los bordes de las islas se equipan con aspersores sectoriales.

- S: Superficie regable y equipada de la UR en $\mathrm{m}^{2}$.

\subsection{Fórmula de la dotación ajustada a la geometría}

Una vez se han calculado los nuevos coeficientes de mayoración por efecto borde de las 78 UR analizadas y sus correspondientes IG con ayuda del software QGIS, el siguiente paso es relacionarlos. Para ello se representan ambas variables enfrentadas en un diagrama de dispersión. El eje $X$ contendrá los valores de los IG y el eje $Y$ los valores de EfB.

Una vez representado dicho diagrama se obtendrá la recta de regresión de los puntos generados, cuya fórmula será la nueva ecuación de efecto borde que se está buscando.

\section{Resultados y conclusiones}

\subsection{Resultados y discusión}

A continuación, se presentan los resultados obtenidos en el estudio. Una vez calculados los coeficientes de mayoración por efecto borde se analizan los resultados del análisis de la geometría.

\subsubsection{Nuevos coeficientes de efecto borde}

Los resultados tras el análisis de conteo de aspersores muestran que, en el sector XXIV, el porcentaje medio de aspersores sectoriales respecto al número total de aspersores es de 29\%, mientras que en el sector XXII-Arga 5 es del 23\%. En ambos casos por debajo del 30-35\% estimado en inicio. No obstante, se ha comprobado que la pluviometría de los sectoriales instalados es un $20 \%$ mayor que la estimada inicialmente. En consecuencia, el valor medio de efecto borde en las UURR analizadas resulta de 2,21 en el sector XXIV y de 1,76 en el sector XXII-Arga 5.

\subsubsection{Análisis de la geometría}


En este apartado se muestran los Índices Geométricos de cada una de las 78 UURR analizadas.

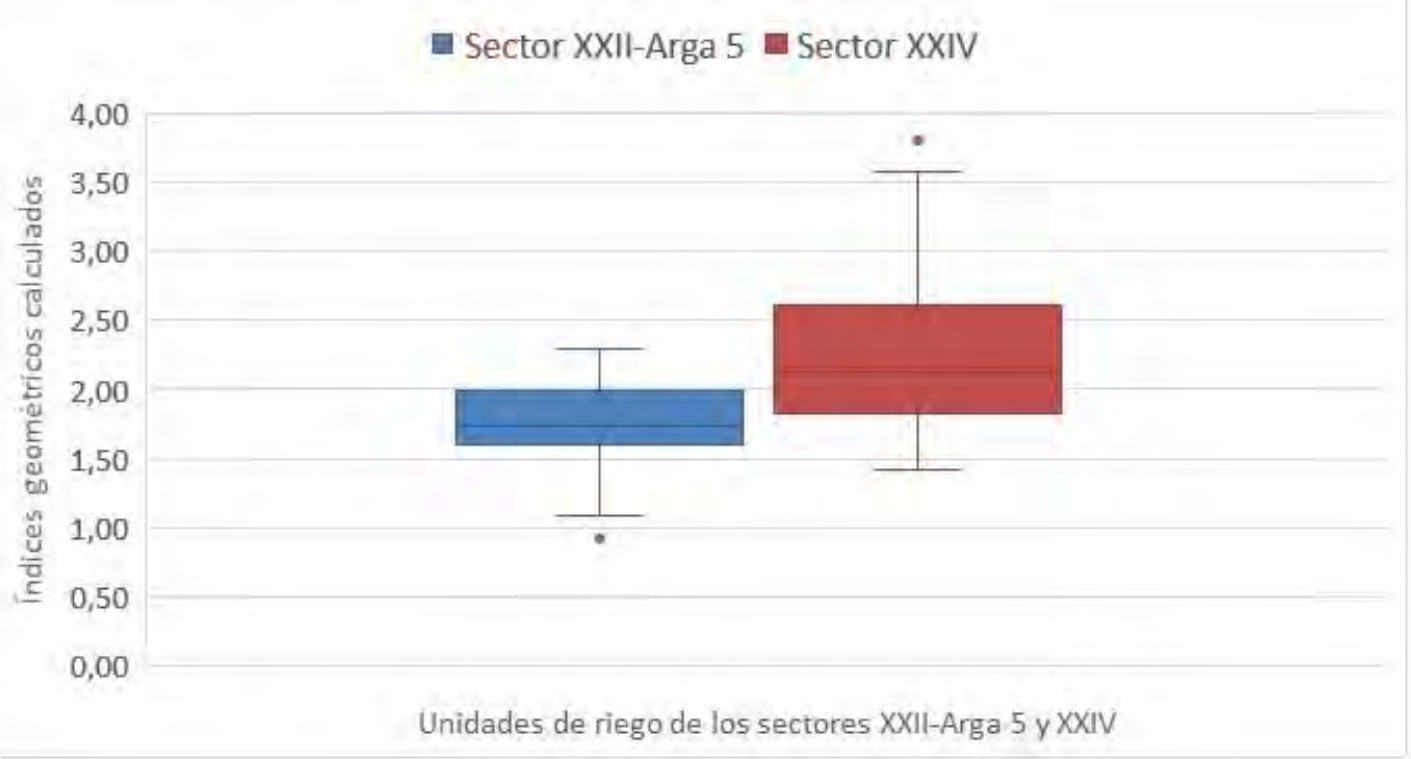

Figura 3: Índices Geométricos calculados para parametrizar la geometría de las UURR analizadas

Como ya se ha comentado, las UURR del sector XXII-Arga 5 tienen una geometría más regular que las del sector XXIV. Como queda reflejado en la Figura 3 , los valores de IG del sector XXIV son mayores, y están más dispersos entre sí. Esto representa la mayor irregularidad y menor homogeneidad de sus UURR.

\subsubsection{Fórmula de la dotación del hidrante ajustada a la geometría de la} UR

Por último, se muestra el diagrama de dispersión (Figura 4) en el que se representan los nuevos coeficientes de efecto borde recalculados frente a los índices geométricos de todas las UURR estudiadas. 


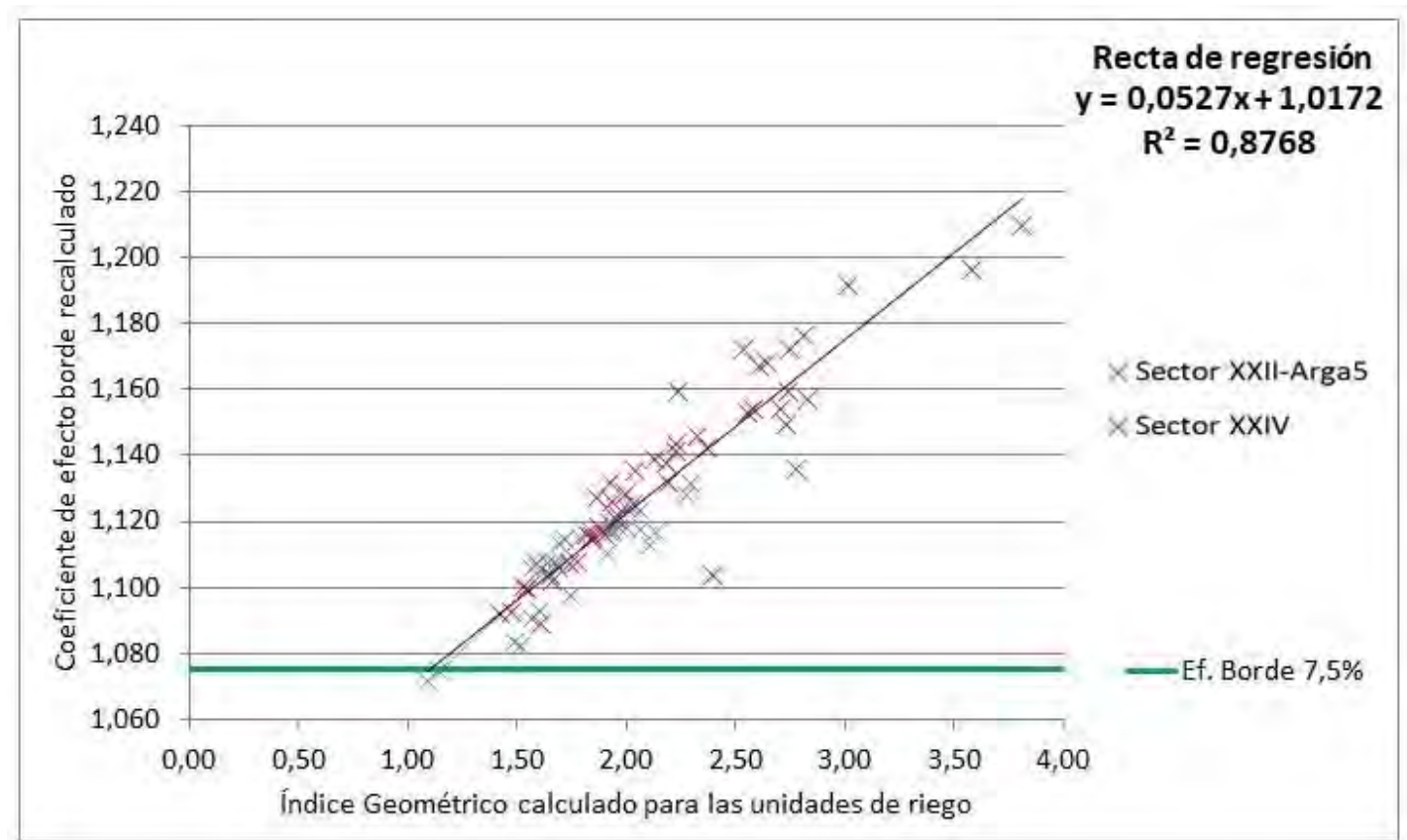

Figura 4: Representación de los nuevos coeficientes de efecto borde de las UURR analizadas frente a sus índices geométricos

Se observa que la correlación es muy alta, con un $\mathrm{R}^{2}$ de aproximadamente 0,9 . Con esta correlación, la ecuación (4) de la recta de regresión generada se entiende que sirve para calcular el coeficiente de mayoración por efecto borde de cualquier UR con cualquier forma.

$$
y=0,0527 x+1,0172
$$

\section{Donde:}

- y: Nuevo coeficiente de mayoración por efecto borde, adimensional.

- $\quad$ x: Índice Geométrico de la UR, adimensional.

Por último, se incorpora la nueva ecuación de efecto borde, que sustituye al valor fijo de mayoración por efecto borde de 1,075, utilizado hasta ahora para el cálculo de las dotaciones de los hidrantes (5).

$$
\mathrm{Q}_{i}(\mathrm{l} / \mathrm{s})=\left(\mathrm{S}_{i} / 7\right) \times(6,67 / 3.600) \times(0,0527 \times 1 \mathrm{Gi}+1,0172)
$$

Donde:

- $\quad S_{i}:$ Superficie de cada unidad de riego, en $\mathrm{m}^{2}$.

- 7: $N^{0}$ de sectores en que de forma general se dividen las unidades de riego.

- 6,67: Pluviometría, en $\mathrm{l} /\left(\mathrm{m}^{2} \times\right.$ hora $)$.

- $\quad \mathrm{IG}_{\mathrm{i}}$ : Índice geométrico de las unidades de riego.

$$
\mathrm{IGi}=(\mathrm{Pi} \times 100) / \mathrm{Si}
$$

Donde:

- IG: Índice Geométrico utilizado para parametrizar la forma de las UR, adimensional. 
- $\quad P_{\mathrm{i}}$ : Perímetro de la UR (en $\mathrm{m}$ ). Cuando una UR tenga en su interior una o más islas de secano (con por ejemplo valores naturales de obligada conservación), sus perímetros se deben sumar al de la UR, ya que los bordes de las islas se equipan con aspersores sectoriales.

- $\quad S_{\mathrm{i}}$ : Superficie regable y equipada de la UR, en $\mathrm{m}^{2}$.

Como resultado final, se comprueba que la fórmula (5) es efectiva. Para ello se representan en otro diagrama de dispersión Figura 5 los caudales calculados con dicha ecuación (dotación de los hidrantes) frente a los que han obtenido los diseñadores de parcela al dimensionar los requerimientos de las UURR.

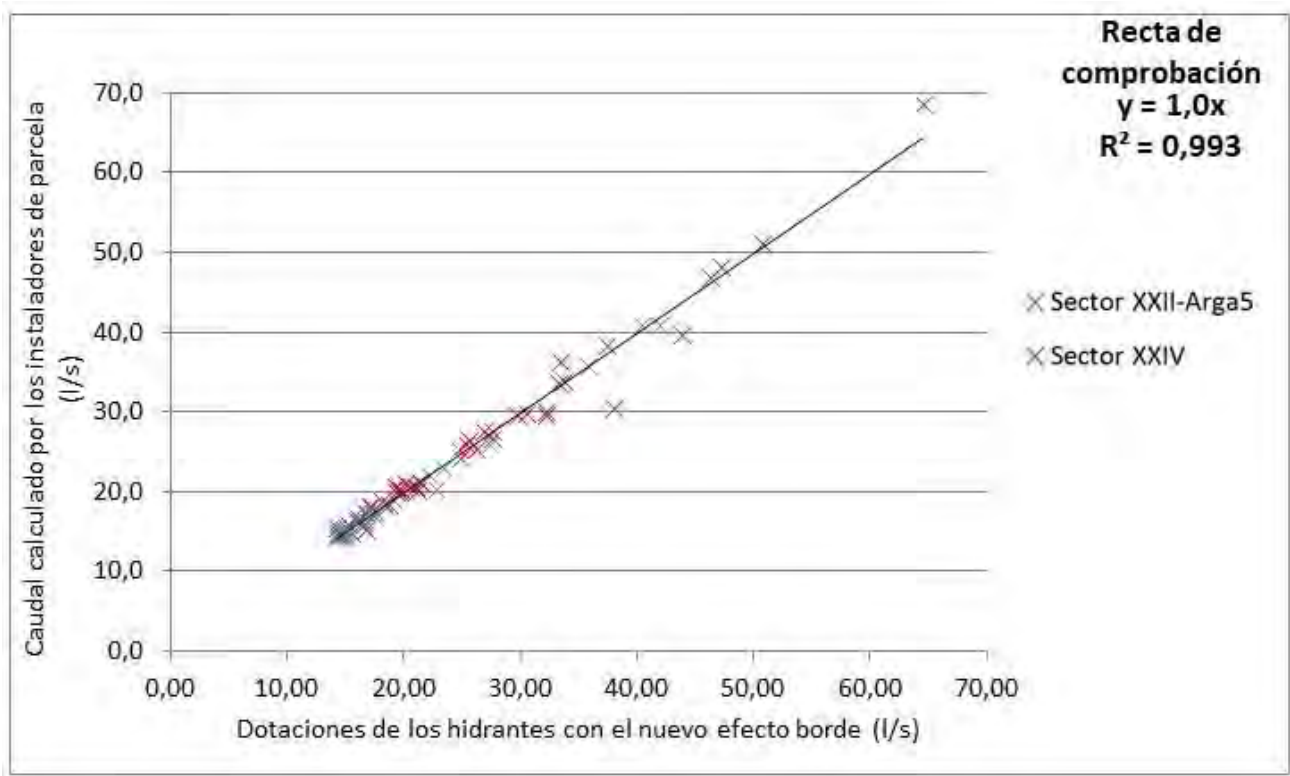

Figura 5: Comparación de los caudales obtenidos con la fórmula de la dotación de los hidrantes y los requerimientos solicitados por los diseñadores de parcela

Tal y como demuestra el $R^{2}$ de aproximadamente 1 , los caudales a suministrar por los hidrantes se ajustarían perfectamente con los requerimientos si se utiliza la fórmula propuesta.

En la revisión bibliográfica realizada, se ha observado que, a pesar de que existen menciones de la importancia de la forma de las parcelas en la eficiencia de los ramales (Arviza et al., 1993) no se ha encontrado ningún autor que añada esta variable a la fórmula del cálculo de la dotación de los hidrantes. Hay ecuaciones (6) similares a la utilizada por INTIA (Planells et al., 1999), que se utilizan para estudios posteriores (Planells et al., 2001), pero no contemplan la geometría de las parcelas en sus cálculos.

$$
d=2,778 \times P_{m s} \times \frac{S}{N_{s}}
$$

Donde:

- $\quad P_{m s}$ es la pluviosidad $\left(1 / m^{2} \times h\right)$ dada por los aspersores.

- S: superficie (ha).

- $\quad N_{s}$ número de subunidades. 
2,778 proviene de la división de $10.000 \mathrm{~m}^{2} /$ ha entre $3.600 \mathrm{~s} / \mathrm{h}$ (García-Prats \& Guillem-Picó, 2016).

\subsection{Conclusiones}

Una vez comentados los resultados, las conclusiones serán las siguientes:

- Es necesario ajustar la fórmula de cálculo de las dotaciones de los hidrantes a un porcentaje y a una pluviometría de aspersores sectoriales más próximos a la realidad instalada.

- El índice geométrico calculado es representativo de la forma que tienen las UR, y por tanto de la proporción de aspersores sectoriales respecto del total con los que se equiparán.

- La fórmula final se adapta a todas las UURR del estudio, ya que el IG tendrá un valor mayor en aquellas con una geometría complicada, y menor en las más regulares, variando así la mayoración de caudal en los bordes frente al valor fijo establecido en inicio para todas las UURR.

- La fórmula final propuesta en este trabajo ajusta con precisión el cálculo de las dotaciones de los hidrantes al caudal que demandan realmente las instalaciones ejecutadas en las UURR.

\section{Recomendaciones}

Una vez analizados los resultados, se puede observar la importancia que tiene ajustar al máximo los valores de cálculo con el fin de aumentar la eficiencia en la aplicación del agua en la unidad de riego. El agua es un bien escaso, y por tanto se debe utilizar de la manera más eficiente posible. Al incorporar la geometría de la UR en el cálculo de las dotaciones de los hidrantes se ajusta de forma más precisa la estimación del caudal a suministrar por los hidrantes a la realidad de los requerimientos de la unidad de riego.

\section{Bibliografía}

Arviza Valverde, J., Santamarina Siurana, C., Guillem-Picó, S., Julia Igual, J. F., \& de Miguel Gómez, M. D. (1993). Curso de redes hidráulicas de riego a presión (DIAZOTEC S). Colegio Oficial de Ingenieros Agónomos de Aragón, La Rioja y País Vasco.

García-Prats, A., \& Guillem-Picó, S. (2016). Adaptation of pressurized irrigation networks to new strategies of irrigation management: Energy implications of low discharge and pulsed irrigation. Agricultural Water Management, 169, 52-60.

Gobierno de Navarra. Ley foral 1/2002, de 7 de marzo, de infraestructuras agrícolas (2002).

Planells, P., Ortega, J. F., Valiente, M., Montero, J., \& Tarjuelo Martín-Benito, J. M. (1999). Criterios para el diseño de redes colectivas de riego. Ingeniería Del Agua, 6(1), 27-36.

Planells, P., Tarjuelo Martín-Benito, J. M., \& Ortega, J. F. (2001). Optimización de estaciones de bombeo en riego a la demanda. Ingeniería Del Agua, 8(1), 39-51. 